\title{
SDSS IV MaNGA-Rotation Velocity Lags in the Extraplanar Ionized Gas from MaNGA Observations of Edge-on Galaxies
}

\author{
D. Bizyaev ${ }^{1,2,3}$, R. A. M. Walterbos ${ }^{4}$, P. Yoachim ${ }^{5}$, R. A. Riffel ${ }^{6,7}$, J. G. Fernández-Trincado ${ }^{8}$, K. Pan $^{1}$, A. M. Diamond-Stanic ${ }^{9,10}$, \\ A. Jones ${ }^{11}$, D. Thomas ${ }^{12}$, J. Cleary ${ }^{13}$, and J. Brinkmann ${ }^{1}$ \\ ${ }^{1}$ Apache Point Observatory and New Mexico State University, Sunspot, NM 88349, USA \\ ${ }^{2}$ Sternberg Astronomical Institute, Moscow State University, Moscow, Russia \\ ${ }^{3}$ Special Astrophysical Observatory of the Russian AS, 369167, Nizhnij Arkhyz, Russia \\ ${ }^{4}$ Department of Astronomy, New Mexico State University, Las Cruces, NM 88003, USA \\ ${ }^{5}$ Department of Astronomy, University of Washington, Seattle, WA 98195, USA \\ ${ }^{6}$ Departamento de Física, CCNE, Universidade Federal de Santa Maria, Av. Roraima, 1000-97105-900, Santa Maria, RS, Brazil \\ Laboratório Interinstitucional de e-Astronomia-LIneA, Rua Gal. José Cristino 77, Rio de Janeiro, RJ-20921-400, Brazil \\ ${ }^{8}$ Institut Utinam, CNRS UMR 6213, Université de Franche-Comté, OSU THETA Franche-Comté-Bourgogne, \\ Observatoire de Besançon, BP 1615, F-25010 Besançon Cedex, France \\ ${ }^{9}$ Department of Astronomy, University of Wisconsin-Madison, Madison, WI 53706, USA \\ ${ }^{10}$ Department of Physics and Astronomy, Bates College, Lewiston, ME 04240, USA \\ ${ }^{11}$ Max-Planck Institute for Astrophysics, Karl-Schwarzschild-Str 1, Garching, D-85748, Germany \\ ${ }^{12}$ Institute of Cosmology and Gravitation, University of Portsmouth, Dennis Sciama Building, Portsmouth PO1 3FX, UK \\ ${ }^{13}$ Department of Physics \& Astronomy, Johns Hopkins University, Bloomberg Center, 3400 North Charles Street, Baltimore, MD 21218, USA \\ Received 2016 September 29; revised 2017 March 21; accepted 2017 March 24; published 2017 April 19
}

\begin{abstract}
We present a study of the kinematics of the extraplanar ionized gas around several dozen galaxies observed by the Mapping of Nearby Galaxies at the Apache Point Observatory (MaNGA) survey. We considered a sample of 67 edge-on galaxies out of more than 1400 extragalactic targets observed by MaNGA, in which we found 25 galaxies (or 37\%) with regular lagging of the rotation curve at large distances from the galactic midplane. We model the observed $\mathrm{H} \alpha$ emission velocity fields in the galaxies, taking projection effects and a simple model for the dust extinction into account. We show that the vertical lag of the rotation curve is necessary in the modeling, and estimate the lag amplitude in the galaxies. We find no correlation between the lag and the star formation rate in the galaxies. At the same time, we report a correlation between the lag and the galactic stellar mass, central stellar velocity dispersion, and axial ratio of the light distribution. These correlations suggest a possible higher ratio of infalling-to-local gas in early-type disk galaxies or a connection between lags and the possible presence of hot gaseous halos, which may be more prevalent in more massive galaxies. These results again demonstrate that observations of extraplanar gas can serve as a potential probe for accretion of gas.
\end{abstract}

Key words: galaxies: ISM - galaxies: kinematics and dynamics - galaxies: spiral - intergalactic medium

\section{Introduction}

Warm, ionized gas at high altitude from the midplane has been detected in the Milky Way (Hoyle \& Ellis 1963; Reynolds 1971; Reynolds et al. 1973, 1999; Shull et al. 2009). It is also observed in other galaxies (Dettmar 1990; Rand et al. 1990; Rand 1997, 2000; Hoopes et al. 1999; Rossa et al. 2004) at distances up to several kiloparsecs (Rossa \& Dettmar 2003; Wu et al. 2014) from the galactic midplane. It is one of the tracers indicating significant amounts of extraplanar gas. This material is envisioned as an important component of galactic evolution that probes disk-halo interaction (Putman et al. 2012). The material may help support and regulate star formation in galaxies by replenishing gas close to the midplane.

The origin of the extraplanar gas is still unclear. It may have contributions from two sources: cosmological accretion from the reservoir of the intergalactic medium (Binney 2005) or gas associated with galactic fountain flows in galaxies that are produced by star formation in the disk (Shapiro \& Field 1976; Bregman 1980; Norman \& Ikeuchi 1989; Marinacci et al. 2011; Fraternali et al. 2013). Both of these effects may be at work together (Haffner et al. 2009; Benjamin 2012). Since the gas flow is either parallel to the sky plane for edge-on galaxies or obscured by gas in the galactic midplane, it is difficult to establish from observations whether the gas is traveling out or in, as caused by fountain flows, or represents some quasistable phase of gas energized by continuous activity in the disk. The quantity and distribution of the material suggests the latter, but this does not imply that a connection to flows is not present. Different classes of objects (e.g., active galactic nuclei, young stars, old evolved hot stars, or dynamical features) can be responsible for the ionization and motion of extraplanar gas. Circulation of the gas between the galaxy and the intergalactic medium may be driven by star formation in the disk.

A deviation of the gas component's rotation from cylindrical at large distances form the midplane, i.e., the rotation velocity lag, or briefly "lag," has been noticed in several nearby galaxies. It has been measured in atomic gas (see e.g., Swaters et al. 1997; Matthews \& Wood 2003; Zschaechner et al. 2011, 2015; Gentile et al. 2013; Kamphuis et al. 2013; Zschaechner \& Rand 2015) and ionized gas (Fraternali et al. 2004; Heald et al. 2006a, 2006b, 2007; Kamphuis et al. 2007, 2011; Rosado et al. 2013; Wu et al. 2014; Boettcher et al. 2016). The number of galaxies with measured rotation velocity lags is still small, and the lack of data hinders statistical studies. It has been known for a while that a strong correlation exists between star formation per unit disk area and the presence of ionized extraplanar gas (Dettmar 2004; Ho et al. 2016), but fewer galaxies have measurements of kinematic lags. An inverse 
correlation between the lag amplitude and the star formation activity in galaxies was suggested by Heald et al. (2007) but not confirmed by Zschaechner et al. (2011). In this paper we define lag as a decrease in rotational velocity with height.

Studying correlations between the lag and general galactic parameters can be a powerful tool for understanding the nature of the extraplanar gas. The most recent results (Zschaechner \& Rand 2015) indicate a radially decreasing lag amplitude in galaxies, which the authors interpret as likely pointing to an internal origin of the lags. Lag measurements can also provide potential evidence for accretion of gas onto galactic disks (e.g., Fraternali \& Binney 2006, 2008). Large integral field unit (IFU) surveys being conducted in recent years (e.g., CALIFA, ${ }^{14}$ Sanchez et al. 2016; MaNGA, ${ }^{15}$ Bundy et al. 2015; and SAMI, ${ }^{16}$ Allen et al. 2015) provide excellent data from panoramic spectroscopy suitable for studying the extraplanar gas component in various types of galaxies. In this paper we employ a growing IFU data set from the MaNGA survey for studying the kinematics of ionized gas around a substantial sample of edge-on disk galaxies.

\section{MaNGA Observations}

MaNGA is a huge IFU spectroscopic survey (Bundy et al. 2015; Drory et al. 2015) that is part of the fourth phase of the Sloan Digital Sky Survey $\left(\right.$ SDSS) ${ }^{17}$ (Blanton et al. 2017). MaNGA plans to deliver spectroscopic maps with $R \sim 2000$ for about 10,000 nearby galaxies by 2020 . MaNGA uses the Sloan $2.5 \mathrm{~m}$ telescope at Apache Point Observatory (Gunn et al. 2006) and spectrographs that cover the spectral range 3600-10300 $\AA$ (Smee et al. 2013). The survey's target selection (Law et al. 2015) and observing strategy provide maps with kiloparsec-scale spatial resolution of the stellar and ionized gas kinematics in the galaxies: the resulting FWHM $\sim 2.5$ arcsec (Law et al. 2016) in the parameter maps corresponds to $1.5 \mathrm{kpc}$ at the median redshift of the survey of 0.03 . The observing strategy provides contiguous coverage of targets, without leaving unobserved gaps. The spectra are fluxcalibrated to the precision of a few per cent (Yan et al. 2016). Current data release MaNGA Product Launch-4 (MPL-4, Law et al. 2016; K. Westfall et al. 2017, in preparation) provides spectral data cubes and various parameter maps derived from them for more than 1400 unique galaxies (including ancillary targets).

\subsection{The Sample of Edge-on Galaxies in MaNGA}

Since all MaNGA galaxies were selected based on SDSS imaging products (Law et al. 2015), we were able to download and inspect the color-composite images of all galaxies from the SDSS SAS ${ }^{18}$ archive (Blanton et al. 2011; Alam et al. 2015). We identified edge-on galaxies from visual inspection similar to how it was done in Bizyaev et al. (2014): objects were classified as edge-on systems if they had no traces of spiral arms that could be seen, and also if the dust lane (if present) was projected on or very close to the galaxy's disk midplane. We also restricted our search to objects not showing obvious signs of interaction. As a result, we identified 67 edge-on

\footnotetext{
$\overline{14}$ Calar Alto Legacy Integral Field spectroscopy Area survey.

15 Mapping Nearby Galaxies at Apache Point Observatory.

16 Sydney-Anglo-Australian Observatory Multi-object Integral field survey.

17 Sloan Digital Sky Survey, http://sdss.org.

18 Science Archive Server.
}

galaxies, without any additional selection by morphological type, size, etc. MaNGA IFUs range in size from $12 \operatorname{arcsec}$ (19 fibers) to 32 arcsec (127 fibers); see the IFU description in Drory et al. (2015). Out of the 67 selected edge-on galaxies, $49 \%$ were observed with the largest, 127-fiber IFUs; for the rest, $9 \%, 24 \%, 13 \%$, and $5 \%$ were observed with IFUs containing 91, 61, 37, and 19 fibers, respectively.

\subsection{Selecting Edge-on Galaxies with Measurable Rotation Velocity Lag}

The MPL-4 release provides radial velocities of gas estimated for different emission lines on a spatial grid of 0.5 arcsec spaxel size. We utilize $\mathrm{H} \alpha$ fluxes with uncertainties to estimate the signal-to-noise ratio $(\mathrm{S} / \mathrm{N})$, and $\mathrm{H} \alpha$ radial velocities with uncertainties for drawing maps of the gas kinematics. In the majority of galaxies the $\mathrm{H} \alpha$ fluxes are detected at altitudes higher than $1 \mathrm{kpc}$ from their galactic midplane. Then we considered the vertical profiles of the radial velocities at different radii from the center, typically in 1-2 kpc wide bins along the major axis. Only the spaxels with good data quality flags and with $\mathrm{S} / \mathrm{N}>3$ were taken into account. We find that 42 objects in the sample show a rather irregular shape of the vertical velocity profiles, whereas 25 galaxies show a regularly decreasing velocity amplitude, i.e., lag, with vertical distance from the midplane, at all radial distances from the center within the limits of our angular resolution of 2.5 arcsec. We refer to this group of galaxies as the subsample in the text below, to distinguish it from the main initial sample of edge-on galaxies.

Figure 1 shows two examples of galaxies with vertical velocity profiles that suggest that the rotation curve has lower amplitude above the midplane than in the midplane, i.e., that we observe a lag. The top panel in Figure 1 is a typical galaxy in our sample, while the bottom panel shows a galaxy with a less clear lag.

We cannot calculate the lag amplitude directly from Figure 1 because of projection effects and possible dust extinction. It is worth noting, however, that these effects would not decrease the amplitude of the rotation curve systematically with altitude above the galactic midplane in the case of pure cylindrical rotation of gas, i.e., in the case of no lag. Thus, we assume that the regular decrease in the gas rotation velocity with vertical distance to the midplane is an indication of a lag. We selected 25 galaxies with regularly decreasing velocity amplitude with vertical distance to the midplane for the further modeling.

Images of selected galaxies are shown in Figure 2. It is interesting to note that the IFU size distribution for this subsample well matches that of the overall sample of 67 MaNGA edge-on galaxies: $54 \%, 13 \%, 21 \%, 8 \%$, and $4 \%$ for IFUs containing $127,91,61,37$, and 19 fibers, respectively. We will come back to the 42 galaxies that do not show a systematic lag in the discussion in Section 4.

The panels in Figure 2 are 1 arcmin on each side. The largest IFU bundle used for 13 of the 25 galaxies is about half that size. Thus, none of the galaxies is fully covered in the radial extent visible in the figure. This fact implies that we probe the lag mostly over the inner disk and cannot use these data to address whether there are radial variations in the lag. At the same time, the data probe well outside the central region and the galactic midplane.

A single fiber corresponds to $1.5 \mathrm{kpc}$ at the median redshift of the survey. The smallest fiber bundle for this sample (19 

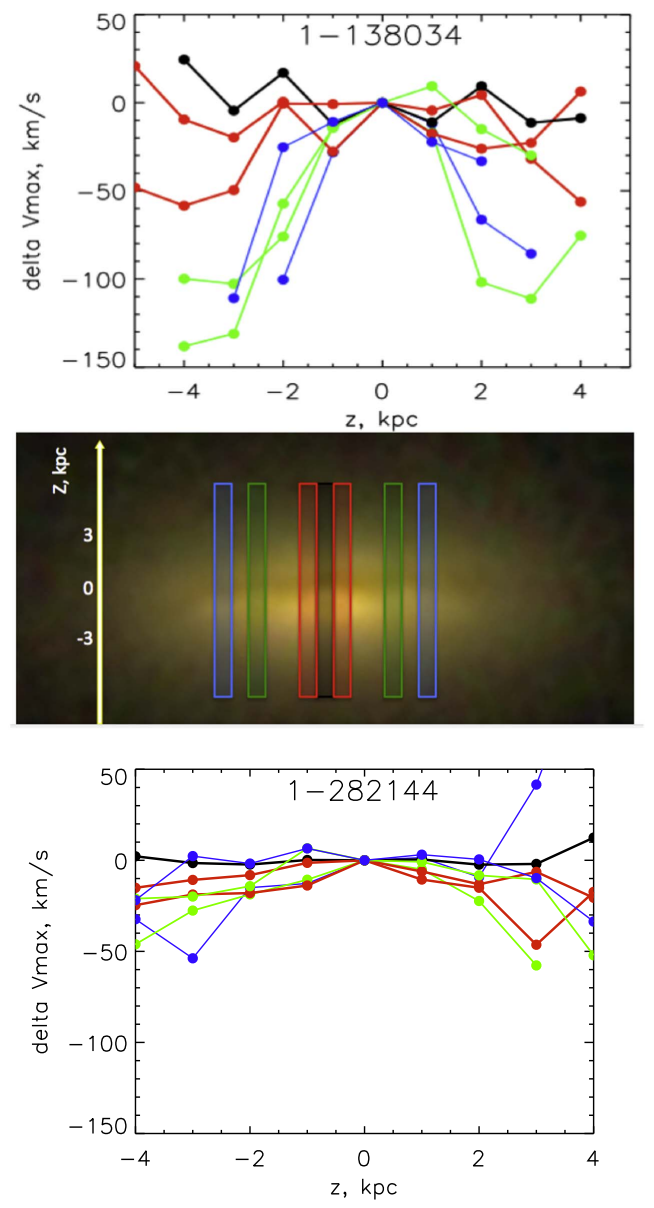

Figure 1. Vertical profiles of radial velocity in two galaxies from our sample. Top panel: the galaxy 1-138034 is shown as a typical example. All velocities are shown with respect to the galactic midplane velocity. The black curve designates the velocity vertical profile that goes through the center $(X=0)$. The red curves are for $X=0.8 \mathrm{kpc}$, the green ones correspond to $X=3 \mathrm{kpc}$, and the blue ones designate $X=4 \mathrm{kpc}$. Here $X$ designates the projected distance to the center along the major axis on the edge-on galaxy. Middle panel: regions used for making the radial velocity profiles in the galaxy 1-138034 designated with the same colors as in the top panel. The background image of the galaxy is taken from the SDSS SAS server (see http://sdss.org). Bottom panel: same vertical profiles of radial velocity as in the top panel shown for the galaxy 1-282144, in which the lag is seen less clearly.

fibers) still has five fibers across each galaxy. The largest bundle used for more than half the subsample has from 11 to 13 fibers across the major axis of each galaxy, depending on the orientation of the fiber bundle. Coverage decreases away from the center of the galaxy, due to the hexagonal shapes of the fiber bundles, so the radial extent over which lags may be probed is somewhat smaller than the extent of the major axis (Drory et al. 2015).

\section{Modeling Velocity Fields of Ionized Gas}

Below we assume that the ionized gas that emits $\mathrm{H} \alpha$ follows a bi-exponential three-dimensional distribution in the galaxy, and introduce its rotation curve as an analytical function. We construct model maps of the two-dimensional distribution of $\mathrm{H} \alpha$ radial velocities by integrating the $\mathrm{H} \alpha$ emission profiles weighted by volume density along the line of sight. Then we compare the resulting $\mathrm{H} \alpha$ velocity fields with observations and obtain the model parameters via chi-square optimization.
The model rotation curve in the midplane is approximated by three parameters: radius $r_{0}$ to which the velocity rises linearly, the rotation velocity at $r_{0}, V_{\max }$, and the gradient of the rotation curve beyond $r_{0}, d v / d r$. The last of these allows for the possibility of a slightly rising or decreasing rotation curve. Hence, $V(r)=V_{\max }\left(r / r_{0}\right)$ if $r<r_{0}$, and $V(r)=V_{\max }+(d v / d r)\left(r-r_{0}\right)$ if $r \geqslant r_{0}$, where $\mathrm{r}$ is the distance to the galactic center.

The lag of the rotation velocity is introduced as a linear vertical gradient $d v / d z: V(r, z)=V(r)-|z| d v / d z$, where $z$ is the vertical distance in kiloparsecs. The rotation curve and the lag are assumed to be independent of the polar angle in the disk plane, $\phi$. Since the observed rotation curve is a result of projection of $V(r, \phi, z)$ on the sky plane, we have to consider the line-of-sight integration of $V(l, z)$ weighted by the volume density of the $\mathrm{H} \alpha$ luminosity $f_{\mathrm{H} \alpha}$. Here $l(r, \phi)$ designates the distance along a particular line of sight.

We assume that the distribution of the $\mathrm{H} \alpha$ luminosity density is a double exponential: $f_{\mathrm{H} \alpha}(r, z)=f_{0} \exp (-r / h)$ $\exp \left(-|z| / z_{0}\right)$, where the scale length $h$, scale height $z_{0}$, and $f_{0}$ are estimated from the $\mathrm{H} \alpha$ maps of the galaxies using the pipeline for structural parameters used in Bizyaev \& Mitronova (2002, 2009) and Bizyaev et al. (2014). The $\mathrm{H} \alpha$ maps are obtained from the fluxes provided by MPL-4.

We also introduce a dust disk embedded in the galactic disk (coplanar with the ionized gas and stellar disks). The dust extinction coefficient $\kappa(r, z)=\kappa_{0} \exp \left(-r / h_{d}\right) \exp \left(-|z| / z_{d}\right)$ is the volume extinction coefficient at the wavelength of $\mathrm{H} \alpha$ at a certain point $(r, z)$ in the disk, $h_{d}$ and $z_{d}$ are the radial and vertical disk scales, and $\kappa_{0}$ is the value of the extinction coefficient in the center. We neglect dust scattering in the sense that we treat $\kappa$ as a volume absorption coefficient and ignore the possibility that scattered light might contribute along a line of sight.

We assume that each point $(r, \phi, z)$ in the model disk produces an emission line profile with a central wavelength that corresponds to its velocity $V(r, z)$ and a peak height proportional to the volume density of luminosity. We assume the same velocity dispersion sigma of $\sigma_{w}=10 \mathrm{~km} \mathrm{~s}^{-1}$ for all local Gaussian profiles at each $(r, \phi, z)$. For the radial velocity profile at each point in the disk, $v_{p}(w, r, \phi, z)$, we assume that $v_{p}(w, r, \phi, z)=V(r, z) \exp \left(-w^{2} / 2 \sigma_{w}\right)$. Here $w$ is the offset of the radial velocity from the peak velocity $V(r, z)$, and $w=0$ at the peak. If $X$ and $Y$ are the radial (along the major axis) and vertical (along the minor one) coordinates in the sky plane, the integrated velocity profile $V_{p}$ can be written

$$
\begin{aligned}
V_{p}(w, X, Y)= & \int_{-L}^{L} v_{p}(w, r(l), z) \cos (\phi(l)) \\
& \times f_{\mathrm{H} \alpha}(r(l), z) e^{-\tau(r(l), z)} d l,
\end{aligned}
$$

where

$$
\tau(r(l), z)=\int_{l}^{L} \kappa(r(l), z) d l,
$$

and $\cos (\phi(l))$ accounts for the projection of the velocity vector on the line of sight, and the integration along the line of sight is toward our position starting from the far side of the disk. The vertical axis $z$ is aligned with the axis of the sky plane $Y$, so $Y=z$. The limits of integration in $L$ correspond to $r=4 h$, by analogy with stellar disks that truncate at their four radial scales, on average. 


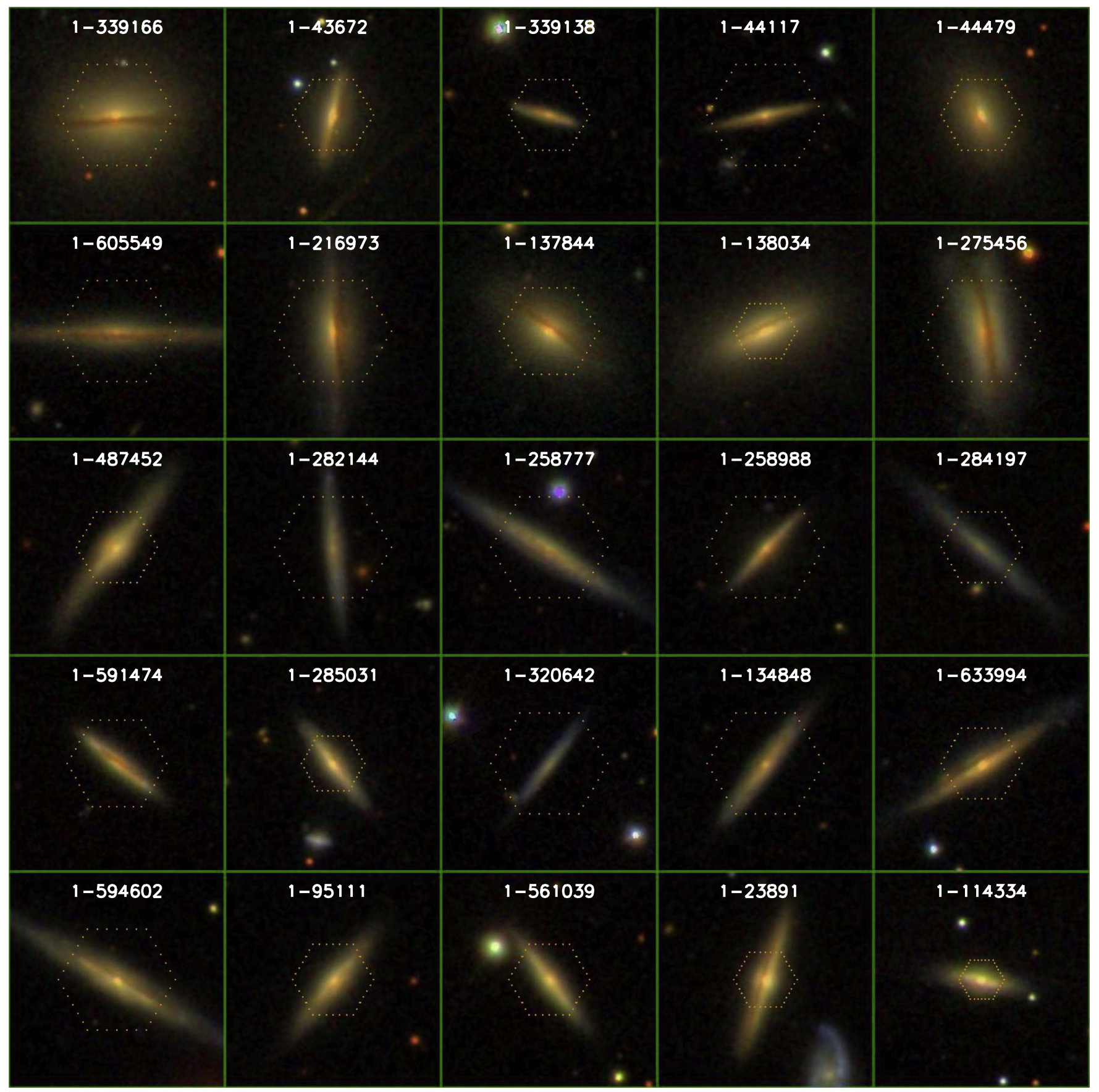

Figure 2. Images of the 25 selected galaxies with regular lag (see Section 2.2). The images are taken from the SDSS SAS server (http://sdss.org). All objects are shown on the same scale: the size of each individual image is 1 arcmin $\times 1$ arcmin. The order of the objects is the same as in Table 1: from the top row left to right corresponds to lines $1,2, \ldots, 5$ in Table 1. The second top row in the figure corresponds to lines $6,7, \ldots, 10$ in Table 1, etc. Table 1 is introduced in Section 3 . The yellow dotted contours show the positioning of MaNGA IFUs on the galaxies.

After the integration along the line of sight we obtain a resulting emission line profile $V_{p}(w)$ of the radial velocity spaced at a set of spaxels $(X, Y)$. The emission line profile is convolved with a Gaussian kernel with FWHM of the spectral resolution. To obtain the final "observable" distribution of the radial velocities in the sky plane, we convolve the $V(X, Y)$ with a twodimensional Gaussian point-spread function (PSF) whose FWHM corresponds to the effective FWHM estimated by the data reduction pipeline for each data cube (Law et al. 2016). Then we estimate the corresponding radial velocity $V(X, Y)$ by fitting the radial velocity profile around the peak velocity value with a Gaussian function.

We estimate the free model parameters via chi-square minimization using the downhill simplex "amoeba" optimization algorithm (Nelder \& Mead 1965). The observing uncertainty of the radial velocity was corrected for the minimum gas velocity dispersion by adding the expected $\mathrm{rms}$ gas velocity, $10 / \sqrt{3} \mathrm{~km} \mathrm{~s}^{-1}$, in quadrature. We also applied spaxel quality flags provided by the pipeline: only spaxels with good data reduction flags and with $\mathrm{S} / \mathrm{N}>3$ were included in 
Table 1

Rotation Velocity Lag and Parameters of our Subsample of MaNGA Galaxies

\begin{tabular}{|c|c|c|c|c|c|c|c|}
\hline MANGA ID & $\begin{array}{l}\text { R.A. (J2000) } \\
\text { (deg) }\end{array}$ & $\begin{array}{l}\text { Decl. (J2000) } \\
\text { (deg) }\end{array}$ & $\begin{array}{c}\mathrm{Lag} \\
\left(\mathrm{km} \mathrm{s}^{-1} \mathrm{kpc}^{-1}\right)\end{array}$ & $\begin{array}{c}e(\mathrm{Lag}) \\
\left(\mathrm{km} \mathrm{s}^{-1} \mathrm{kpc}^{-1}\right)\end{array}$ & $\begin{array}{l}z_{0}(\mathrm{H} \alpha) \\
(\mathrm{kpc})\end{array}$ & $\begin{array}{c}h(\mathrm{H} \alpha) \\
(\mathrm{kpc})\end{array}$ & $\begin{array}{c}\log \left(L(\mathrm{H} \alpha) / L_{\odot}\right) \\
(\operatorname{dex})\end{array}$ \\
\hline $1-339166$ & 116.389689 & 45.772328 & 28 & 8 & 1.3 & 2.5 & 6.1 \\
\hline $1-43672$ & 116.787163 & 42.078716 & 16 & 7 & 1.0 & 3.0 & 5.9 \\
\hline $1-339138^{a}$ & 117.318153 & 46.205040 & 11 & 6 & 0.9 & 1.9 & 6.4 \\
\hline $1-44117$ & 118.863856 & 44.029330 & 13 & 6 & 1.2 & 4.2 & 5.9 \\
\hline $1-44479$ & 119.822532 & 42.008537 & 34 & 9 & 1.5 & 1.5 & 6.3 \\
\hline $1-605549$ & 134.284434 & 51.471546 & 4 & 6 & 1.0 & 6.1 & 5.4 \\
\hline $1-216973$ & 135.833415 & 40.464713 & 27 & 8 & 2.8 & 6.4 & 6.5 \\
\hline $1-137844$ & 139.427012 & 44.100687 & 48 & 11 & 1.4 & 5.4 & 5.6 \\
\hline $1-138034$ & 144.846118 & 47.126864 & 35 & 9 & 0.9 & 2.8 & 6.3 \\
\hline $1-275456$ & 159.382717 & 43.653764 & 14 & 6 & 1.3 & 1.6 & 6.2 \\
\hline $1-487452$ & 166.631358 & 22.777630 & 40 & 10 & 0.8 & 1.2 & 6.1 \\
\hline $1-282144$ & 184.592510 & 46.155351 & 11 & 6 & 1.8 & 8.8 & 6.7 \\
\hline $1-258777$ & 186.394835 & 45.431737 & 22 & 7 & 1.1 & 3.5 & 6.4 \\
\hline $1-258988$ & 187.722485 & 45.219432 & 12 & 6 & 2.1 & 3.4 & 6.7 \\
\hline $1-284197$ & 194.885392 & 42.758926 & 17 & 7 & 1.2 & 3.3 & 5.8 \\
\hline $1-591474$ & 197.580687 & 47.124056 & 14 & 6 & 1.2 & 2.5 & 6.1 \\
\hline $1-285031$ & 198.701372 & 47.351547 & 33 & 9 & 1.0 & 3.0 & 6.5 \\
\hline $1-320642$ & 214.375198 & 47.714958 & 9 & 6 & 1.1 & 4.8 & 6.2 \\
\hline $1-134848$ & 244.331994 & 43.479672 & 12 & 6 & 2.3 & 7.3 & 6.8 \\
\hline $1-633994$ & 247.419951 & 40.686954 & 22 & 7 & 1.4 & 11.0 & 5.9 \\
\hline $1-594602$ & 249.471810 & 39.249823 & 54 & 12 & 1.8 & 10.0 & 6.1 \\
\hline $1-95111$ & 250.668078 & 40.169087 & 29 & 8 & 1.4 & 6.0 & 6.1 \\
\hline $1-561039^{\mathrm{a}}$ & 258.271095 & 35.268616 & 24 & 8 & 0.7 & 1.0 & 5.8 \\
\hline $1-23891$ & 260.228215 & 57.097956 & 38 & 10 & 0.9 & 4.5 & 5.6 \\
\hline $1-114334^{\mathrm{a}}$ & 324.259708 & 11.906203 & 73 & 16 & 0.7 & 4.3 & 5.8 \\
\hline
\end{tabular}

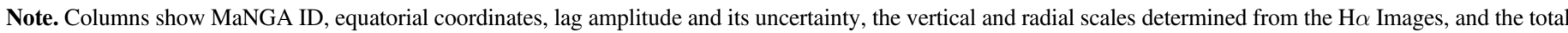
$\mathrm{H} \alpha$ luminosity

${ }^{\mathrm{a}}$ Three galaxies with inclination angles less than $85^{\circ}$ are excluded from the figures with the lag in this paper, see the text.

the minimization. The free parameters of the model are $V_{\max }, r_{0}$, $d v / d r, d v / d z, \kappa_{0}$, and also the central radial velocity $V_{0}$. We do not expect any degeneration between the parameters, so we did not apply any observational constraints to them except a natural requirement of the positivity of $V_{\max }, r_{0}$, and $\kappa_{0}$. We fixed the structural parameters of the dust disk as $z_{d}=z_{0}$ and $h_{d}=h$, which is equivalent to the assumption of uniform mixing of the ionized gas and dust. For comparison, we ran the same modeling for the cases of the volume density of uniformly distributed ionized gas along $r$ and $z$. Also we considered shorter vertical and longer radial scales of the dust, $z_{d}=0.5 z_{0}$ and $h_{d}=1.5 h$, and also $h_{d}=2 h$ and $h_{d}=h$ (following Matthews et al. 1999; Xilouris et al. 1999; Kylafis et al. 2001; Yoachim \& Dalcanton 2006; Bianchi 2007). In all cases considered the resulting model parameters were within $15 \%$ of the original model, which suggests low sensitivity of the output to the input assumptions on the distribution of ionized gas and dust. The lag values derived from the model fits are shown in Table 1.

Figure 3 shows the best-fit model velocity field for the same galaxy shown in Figure 1 for the case of models with no lag and with lag (top and middle panels respectively). The model simultaneously fits lags in the "left" and "right" parts of the galaxy (with respect to the minor axis), so the absolute values of the radial velocity are shown for clarity of presentation. The observed velocity field strongly favors the case with the vertical lag of the rotation curve.

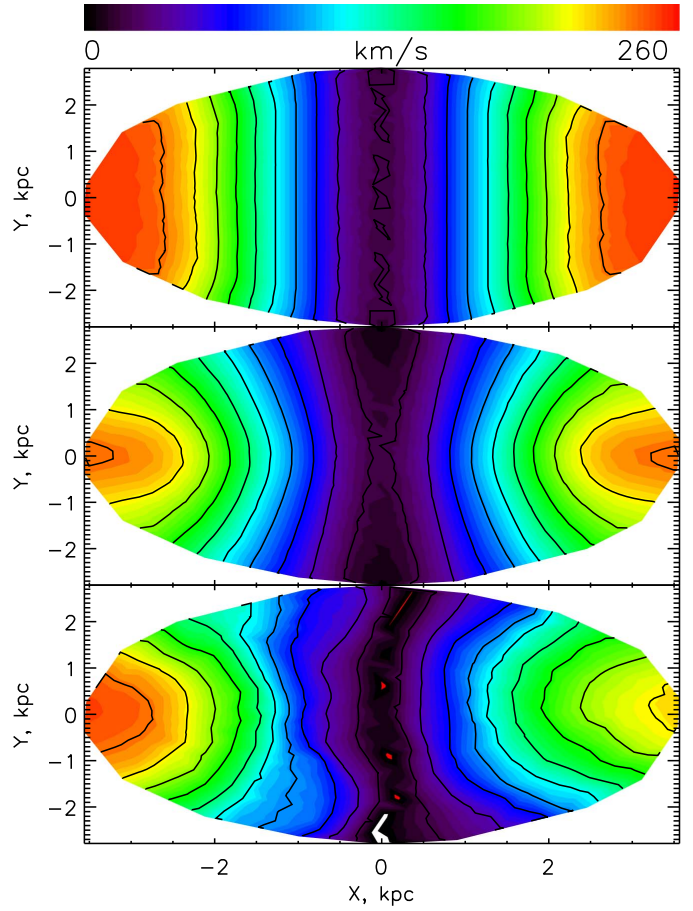

Figure 3. Absolute values of velocity fields of our typical galaxy 1-138034: model field in the case of fitting with no vertical lag (top), model field with lag (middle), and the observed velocity field (bottom). 


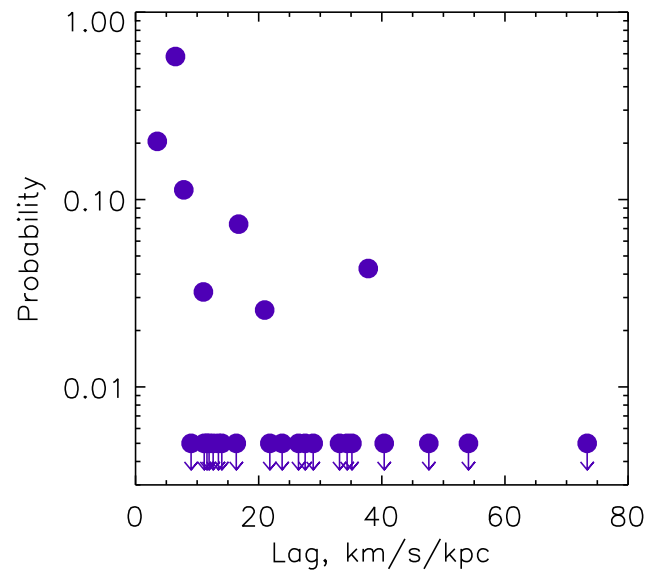

Figure 4. Probabilities of the null hypothesis that models with and without a lag in rotation velocity would statistically result in the same chi-square values (i.e., that chi-square does not decrease enough to justify the additional parameter to model a lag). A few models with small lags show a high probability for no difference, while the bulk of the models gain from using the lag as an additional free parameter. The smallest values of the probabilities are shown as upper limits at $0.5 \%$ for clarity in the figure.

\subsection{Robustness of the Modeling}

To estimate the robustness of our results, we ran the same models with zero fixed-lag term, $d v / d z=0$. Then we performed $F$-tests using the same sample of galaxies modeled with and without lags. Figure 4 shows the probability that chisquare values are significantly similar between the lag and nolag models. Note that most of the probability values are very low, so we truncated the values in Figure 4 at $0.5 \%$ for better presentation. As expected, for several galaxies in the subsample with small lags $\left(10 \mathrm{~km} \mathrm{~s}^{-1} \mathrm{kpc}^{-1}\right.$ and less) the chi-squares do not change significantly, whereas models of the galaxies with large lags fit much better when a vertical gradient of rotation velocity is introduced.

We also constructed a set of synthetic galaxies with typical parameters $h, z_{0}, h_{d}, z_{d}, \kappa_{0}, V_{\max }, d v / d r$, and $d v / d z$. For each case the distribution of radial velocity projected on the sky plane and convolved with the observed PSF was derived. We added normally distributed uncertainties to the radial velocities in each spaxel. We also added typical noise to the fluxes in each spaxel. For the latter we used the completion criterion for MaNGA observations from the science requirement document (Bundy et al. 2015; Law et al. 2015), which states that $\mathrm{S} / \mathrm{N}=5$ has to be obtained at $1.5 R_{e}$ (effective radii) for all program galaxies. We considered a set of noise levels scaled by $\mathrm{S} / \mathrm{N}=1,2,3,5$, and 10 at $1.5 R_{e}$. For each set of galactic parameters and noise levels, we made 30 synthetic galaxies and estimated their parameters with the pipeline described above.

We find that the lag gradient $d v / d z$ is one of the most reliable parameters estimated by the minimization. Its typical uncertainty is $10 \%-15 \%$. When we convert this uncertainty to the actual lag in velocity $d V / d z=(d v / d z) V_{\max }$, the uncertainty in the amplitude of the rotation curve increases the uncertainty in the lag to $20 \%$. The other reasonably estimated model parameters are the radial velocity gradient $d v / d r$ and $V_{\max }$. The value $r_{0}$ has uncertainty of $15 \%$, but in some cases it is shifted systematically from the true initial value in different directions for different model parameters, which increases the uncertainty of this parameter to $25 \%$. The least reliable parameter is the dust absorption coefficient $\kappa_{0}$, which is often
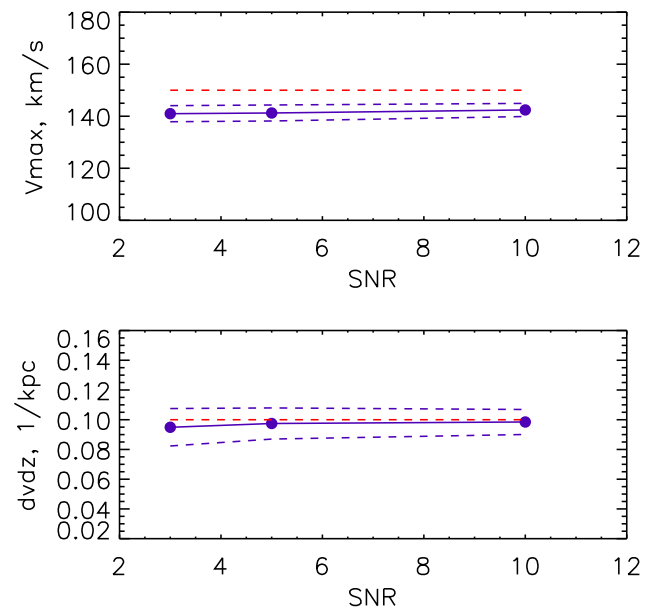

Figure 5. The input (red dashed line) and the output model parameters (blue curves) estimated for the set of synthetic galactic images after inserting noise into them; see text. The solid curves with bullets designate the mean parameters, and the dashed blue curves show $1 \sigma$ standard deviation of the parameters in the set of models with the same $S / N$. The ranges of the vertical axes correspond to typical values of the parameters in our modeling.

underestimated in the modeling, and its uncertainty sometimes reaches $100 \%$. Nevertheless, it does not affect the inferred lag of the rotation curve because the high-altitude regions in the galaxies, which are good tracers of the lag, are less affected by the dust extinction. Figure 5 shows a result of the modeling for a typical set of the model parameters. We conclude that the uncertainty of our estimation of the lag in rotation velocity is $20 \%$. We are also inclined to add a low threshold of $6 \mathrm{~km} \mathrm{~s}^{-1} \mathrm{kpc}^{-1}$ to this uncertainty based on Figure 4, which suggests that, given the uncertainty and resolution of the data, the modeling procedure does not seem sensitive to variations of the lag below $6 \mathrm{~km} \mathrm{~s}^{-1} \mathrm{kpc}^{-1}$.

\subsection{Inclination Effects}

Since our modeling assumes $90^{\circ}$ inclination of galactic disks to the line of sight, any deviation from the perfect edge-on inclination may affect parameters estimated in any studies of edge-on galaxies. de Grijs et al. (1997) showed that the photometric vertical scale height increases by $20 \%$ if the inclination $i$ deviates by $5^{\circ}$ from $90^{\circ}$. We use the visibility of the dust lane in SDSS images of all selected galaxies with lags and the simple technique described in Bizyaev \& Kajsin (2004) and Mosenkov et al. (2015) to estimate the inclination. We do not see any dependence of the lag on the inclination. We find that only three galaxies have $i<85^{\circ}: 1-561039,1-339138$, and $1-114334$. The last of these has $i \approx 82^{\circ}$, and we excluded it from further analysis. The galaxies 1-561039 and 1-339138 have $i$ between $84^{\circ}$ and $85^{\circ}$. Although they do not change trends in figures with the lag shown below in this paper, we excluded them from the figures.

In order to estimate how large the projection due to nonedge-on orientation is in the galaxies, we compare the "projection size" in the galaxies (explained below in this paragraph) with the spatial resolution of MaNGA data cubes. The latter is assumed to be FWHM/2.35, which corresponds to the spatial "sigma" resolution in the MaNGA images, see Section 2). The "projection size" is calculated as the diameter of the galactic disk in projection on the sky plane due to the inclination. We find that the overlap between the near and far sides of galactic disks is less than the spatial resolution in our 
data for all objects. Thus we conclude that the inclination effects can be ignored for our subsample. In general, the inclination effects are important and have to be considered when subsamples of edge-on galaxies are formed from the future MaNGA sample.

\section{Results and Discussion}

We started with a sample of 67 galaxies and found that 25 show systematic patterns in the velocity field that suggest a lag is present. Note that we are not claiming that the other 42 galaxies do not show any lag, only that upon inspection of the velocities they appear less regular and therefore less suitable for the modeling approach we have adopted in this paper.

Previous work in measuring lags for ionized or neutral atomic gas has involved smaller samples of galaxies. Ours is the first study of a moderately large sample observed with one particular IFU setup and analyzed in the same way. The large sample offers opportunities for investigating trends in lags with integral galactic parameters described below.

The lags are expected as a result of galactic fountain flows, but ballistic models of such flows indicate much smaller lags than observed (Collins et al. 2002; Fraternali \& Binney 2006). An inverse correlation between the lag amplitude of ionized gas and the star formation activity in galaxies was suggested by Heald et al. (2007). The inverse correlation was not seen in the neutral gas phase (see Zschaechner \& Rand 2015, and references therein). Surprisingly, no clear trends have been found between lag and other galactic properties so far. In particular, lags have not been seen to correlate with star formation rate, strength of gravity, or environment. The most recent results (Zschaechner \& Rand 2015; Zschaechner et al. 2015) point to a radially decreasing lag amplitude in the neutral gas in galaxies, which the authors interpret as likely indicating an internal origin for the lags. Our radial coverage is limited so we cannot yet address radial variations, but here we consider correlations with star formation rates and global galactic properties for our subsample of galaxies with lag.

To characterize the star formation rate (SFR) and its surface density, we considered the integrated WISE band W4 $(22 \mu \mathrm{m})$ luminosities for the two subsamples. This WISE band has been shown to accurately track the SFR (e.g., Relaño \& Lisenfeld et al. 2007; Zhu et al. 2008; Jarrett et al. 2013) in H II regions and galaxies. The $\mathrm{H} \alpha$ luminosities would underestimate the total SFR due to extinction and lack of coverage of the outer disk in the IFU spectra. We discuss both effects below for the subsample of 25 . The two subsamples showed similar distributions in both total SFR and SFR/area.

We also considered the distribution of axial ratios $(b / a)$ provided in the NASA-Sloan Atlas of galaxies (NSA) for the galaxies (here we use the axis ratio $b / a$ from Stokes parameters at $50 \%$ light radius). Interestingly, all galaxies, while clearly edge-on or close to edge-on, show a full range in $b / a$ from 0.3 to 0.9 . There is a difference in the histogram of $b / a$ : the subsample of 42 shows a peak around $b / a$ of 0.4 , which is not seen in the subsample of 25 . This difference suggests that the fraction of galaxies with less prominent bulge/spheroidal components is larger in the sample of 42 galaxies without regular lags.

We will now analyze trends for the subsample of galaxies where we derived a robust lag value. Figure 6 shows the relationship between the total $\mathrm{H} \alpha$ luminosity and the lag. The luminosity is estimated by integration of the total $\mathrm{H} \alpha$ flux
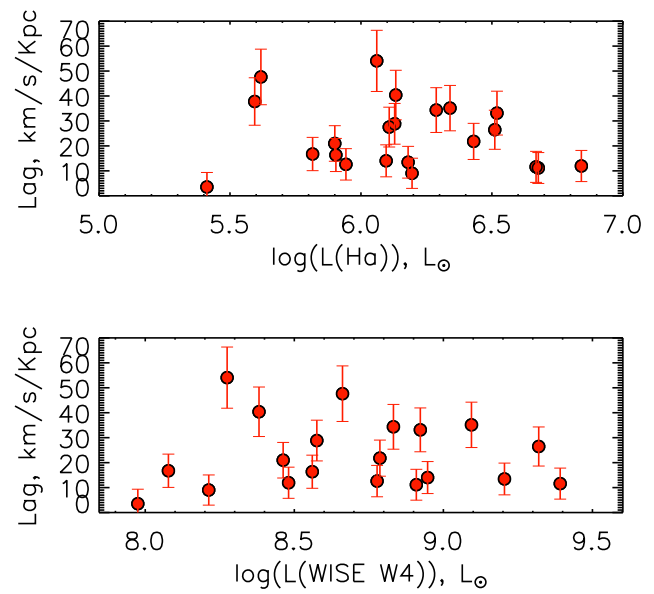

Figure 6. Top: the total $\mathrm{H} \alpha$ luminosity in the galaxies and the lag of the rotation curve. The luminosity is not corrected for internal extinction. Bottom: lag vs. the luminosity of the galaxies in the WISE band W4. The W4 luminosity should correlate well with the star formation rate in the galaxies.

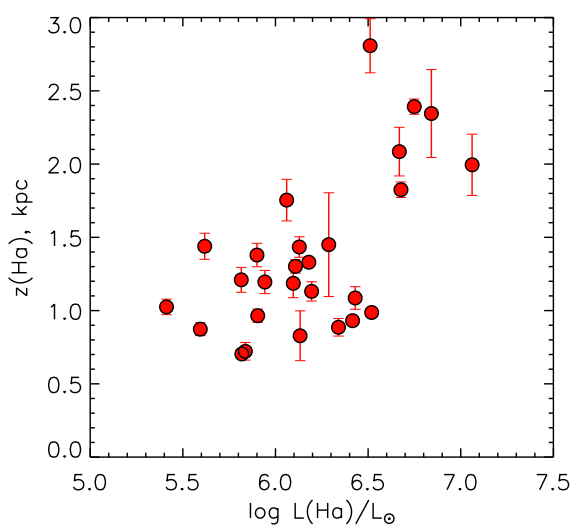

Figure 7. Vertical scale height of the ionized gas vs. the total $\mathrm{H} \alpha$ luminosity in the galaxies.

in the galaxies from MaNGA data. As mentioned above, this estimate may be incomplete due to IFU coverage and it will certainly suffer from extinction. We see no clear correlation (with negative correlation coefficient $\mathrm{cc}=-0.17$ ). The lack of correlation was also apparent when we replaced the $\mathrm{H} \alpha$ luminosity with its mean surface density ( $\mathrm{H} \alpha$ luminosity divided by the disk area, where the disk area was derived using the scale length of the $\mathrm{H} \alpha$ disk; figure not included).

A better indicator of global star formation in the case of edge-on galaxies is the infrared (IR) luminosity and in particular the WISE band W4 luminosity, as discussed above. We calculated the IR luminosity in the WISE band W4 (at $22 \mu \mathrm{m})$, which is very likely optically thin. The WISE fluxes for the galaxies are taken from the ALLWISE catalog (Wright et al. 2010). We find that the $\mathrm{H} \alpha$ and $\mathrm{W} 4$ luminosities are linearly correlated, although with significant scatter. The average ratio between $\mathrm{W} 4$ and $\mathrm{H} \alpha$ luminosities for this subsample is about ten times larger than what is seen in faceon galaxies, consistent with larger extinction of the $\mathrm{H} \alpha$ emission in edge-on systems and with incomplete $\mathrm{H} \alpha$ coverage.

The bottom panel in Figure 6 indicates that there is no correlation ( $\mathrm{cc}=-0.04$ ) between the rotation velocity lag and the star formation rate traced by the IR luminosity, a result similar to the $\mathrm{H} \alpha$ data. The lack of a clear correlation between 

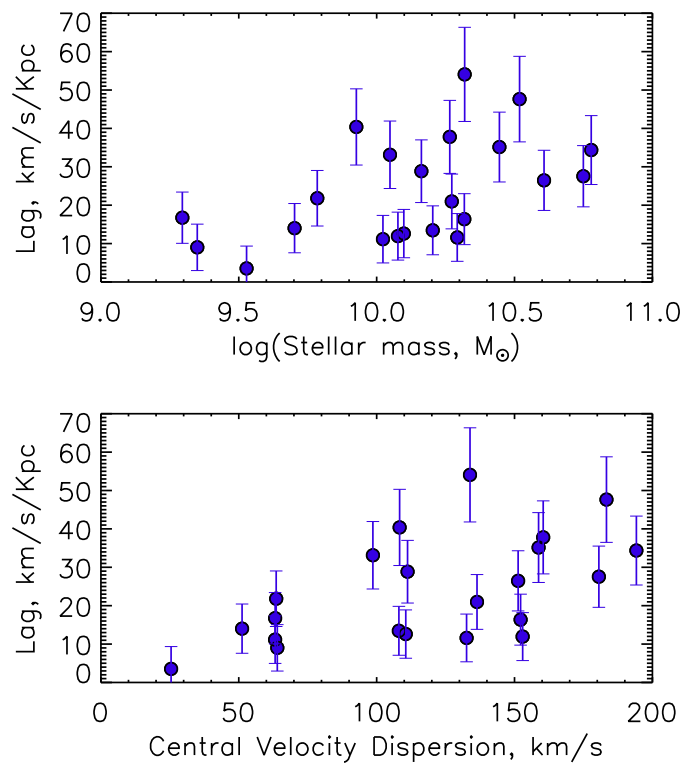

Figure 8. Top panel: the lag of the rotation curve vs. the stellar mass estimated in the NSA. Bottom panel: the lag of the rotation curve vs. the central velocity dispersion in the galaxies.

the star formation rate and the lag amplitude is not necessarily an indicator that there must be an external source of ionization of the gas at high altitudes. We observe a correlation $(\mathrm{cc}=0.55)$ between the vertical scale heights of the ionized gas and the star formation rates, as probed by the total $\mathrm{H} \alpha$ luminosity; see Figure 7. This correlation suggests that internal galactic sources likely play a large role in ionizing gas at high altitudes, consistent with previous studies.

Figure 8 demonstrates a noticeable correlation between the lag and the stellar mass of galaxies $(\mathrm{cc}=0.50)$. The mass in the upper panel is the stellar mass estimated in the $\mathrm{NSA}^{19}$. The lower panel in Figure 8 shows the central stellar velocity dispersion from the NSA $(\mathrm{cc}=0.55)$. Both values are estimated for the NSA from earlier SDSS data, independent of MaNGA. The stellar mass is expected to correlate strongly with the total mass of the dark matter halo (e.g., Behroozi et al. 2013). We also observe significant correlation between the lag and the maximum rotation velocity in the galaxies $V_{\max }$ (cc $=0.55$, not shown here). The latter is expected: lags of large amplitude are easier to detect in galaxies with circular velocities much larger than the total lag.

We considered several additional global parameters of the galaxies in the analysis. A correlation between the lag value and the axial ratio of the starlight for the galaxies (estimated in the NSA) can be seen in Figure $9(\mathrm{cc}=0.48)$. Less significant is correlation with the NSA Sérsic index of the galaxies $(\mathrm{cc}=0.26)$. Both parameters are relevant to the morphological type of galaxies, and suggest that we tend to observe the largest lag values in early-type disk galaxies.

Active galactic nuclei (AGNs) could affect our results by contributing to the $\mathrm{H} \alpha$ luminosity by adding a component not relevant to star formation, as well as contributing to the motion and ionization status of extraplanar gas in the central regions. The former factor can be neglected: we excluded the central regions of 3 arcsec diameter from the calculation of the $\mathrm{H} \alpha$ luminosity and observed the same trends as in Figure 8, which

\footnotetext{
${ }^{19}$ http://nsatlas.org
}
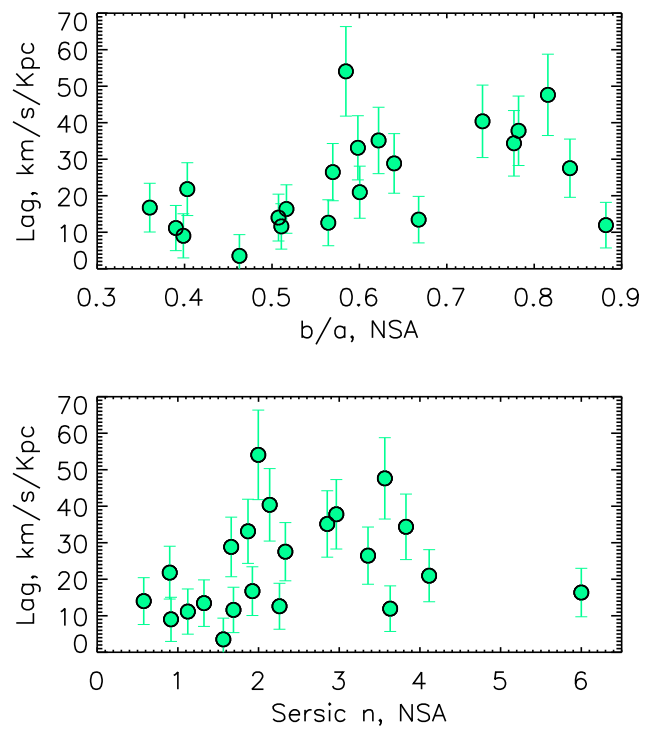

Figure 9. Top panel: the lag of the rotation curve vs. the flatness of the galaxy, as estimated by the NSA. Bottom panel: the lag of the rotation curve vs. the galactic Sérsic index from the NSA.

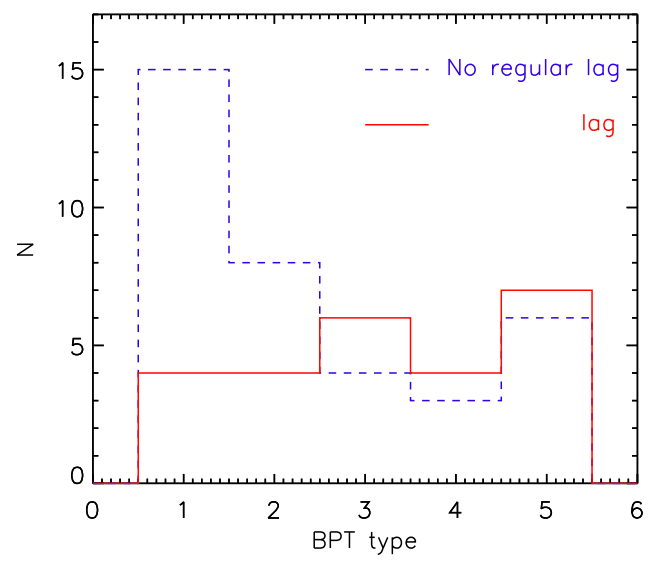

Figure 10. The BPT types determined from spectra of the central regions in galaxies by the MPA-JHU catalog (see the text). The blue dashed curve shows the distribution of galaxies without regular rotation lags, while the red solid red line is that for galaxies with regular lags in the extraplanar ionized gas. The BPT types 1-2 indicate the star-forming galaxies, while types 4-5 designate AGN/LINER classes (see text for detail).

is not surprising since the mean ratio of the excluded luminosity to the total is only 0.04 in our galaxies, and an AGN would likely not be directly seen in an edge-on system.

Most of our galaxies from both subsamples (with and without regular lags) were classified with "BPT types" (named after Baldwin, Phillips, and Terlevich, Baldwin et al. 1981) in the MPA-JHU catalog ${ }^{20}$ (see, e.g., Brinchmann et al. 2004). Note that the classification was made with SDSS spectra, which were obtained from the central 3 arcsec region in galaxies. The BPT types are: 1-star-forming, 2-low signal-to-noise starforming, 3-composite, 4-AGN (excluding LINERs), and 5 -LINER. To summarize them, 1-2 describe the central galaxies dominated by star formation, while 4-5 designate the presence of nuclear activity. Figure 10 demonstrates the distribution of the galaxies with regular lags and those without a regular lag by BPT type. We see that both galaxy subsamples

\footnotetext{
${ }^{20}$ A collaborative project that involved Max-Planck Institute for Astronomy and Johns Hopkins University.
} 


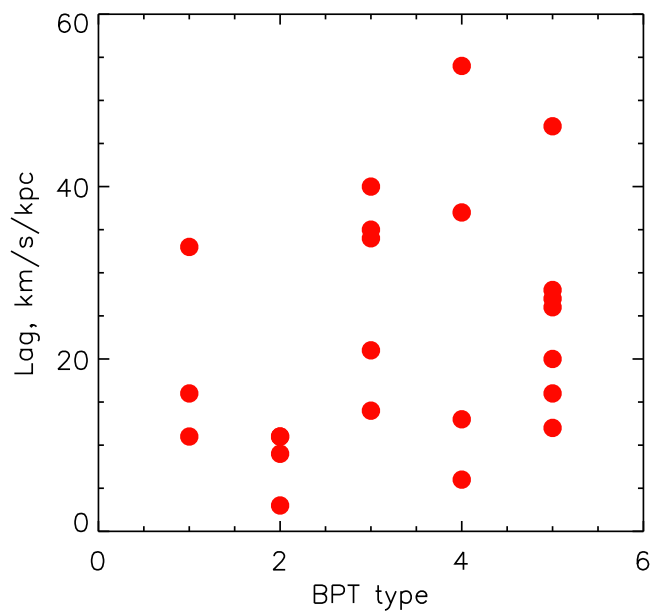

Figure 11. The rotation lag vs. the BPT type. The latter was determined by the MPA-JHU catalog in the central 3 arcsec region.

contain all BPT types, but a larger fraction of the galaxies that do not show a regular lag are dominated by star formation. While this difference seems suggestive of a role for an AGN, we note that BPT types for edge-on systems are likely inaccurate due to extinction. The trend here is likely more consistent with the difference noted earlier in axial ratios for the two subsamples: the subsample without a regular lag includes more pure disk galaxies, which are less likely to have AGN or LINER-type emission in their central region. It may also be simply related to the trend with stellar mass: more massive galaxies are more likely to have an AGN. While the composite and AGN/LINER galaxies all show regular lags, the galaxies dominated by star formation show mostly no regular lags.

Lastly, we considered the distribution of derived lags with the range in radius over which the fits were executed. We looked at both the linear range in kiloparsecs and the scaled range in radius normalized by derived radial scale length. For the 25 galaxies, the range in radius over which the fits were done is between 3 and $20 \mathrm{kpc}$. This large range arises as a result of various factors: the spread in distance for the galaxies, their intrinsic disk sizes, and the size of the IFU bundles. This variety makes it difficult to interpret trends. The one factor that stood out is that the largest lags (over $30 \mathrm{~km} \mathrm{~s}^{-1} \mathrm{kpc}^{-1}$ ) were found only for galaxies for which the total fitting radius was less than $10 \mathrm{kpc}$. It is possible that this trend is consistent with the general decrease found in vertical lags with distance from the center for the $\mathrm{HI}$ distribution across single galaxies (Zschaechner \& Rand 2015). That is, for galaxies that are large enough and well enough covered by the IFU such that the radial range of our fits extends out to between 10 and $20 \mathrm{kpc}$ on average, we find lower overall lag values than for galaxies where the fit range is smaller than $10 \mathrm{kpc}$ in radius. This trend, however, is not seen when the radial range of the fit is expressed in radius normalized by the scale length of the disk. We will need a larger sample and to more deeply consider the coverage of the IFU versus total disk extent of the galaxies to probe such trends further.

The lag amplitude does not correlate strongly with the BPT type, as we show in Figure 11, although it can be noticed that the largest lags in our subsample are observed in the galaxies with high BPT classification, and hence potential AGN activity, while small lags (under $20 \mathrm{~km} \mathrm{~s}^{-1} \mathrm{kpc}^{-1}$ ) occur equally often in star-forming and in mild AGN/LINER galaxies.

\section{Summary and Conclusions}

To summarize, we observe a correlation of the lag amplitude with the stellar mass (and therefore likely also with halo mass), with the central velocity dispersion, and also with galaxy type traced by the flatness of the stellar disk and its global Sérsic index. We see no correlation between the lag and the star formation activity in galaxies in the plots of the lag versus the $\mathrm{H} \alpha$ and IR luminosity in Figure 6. According to Figure 9, latetype disk galaxies tend to have smaller lags, and they show no regular lags more often than the early-type disk systems. It is plausible that larger lags in early-type disk galaxies with higher mass may be related to the higher infalling-to-local gas ratio in these systems compared to lower-mass galaxies. Such accreting gas may come from the intergalactic medium (IGM) or, alternatively, such massive galaxies are more likely to have prominent intrinsic hot gaseous halos that may interact with the disk-halo gas to produce lagging velocities. Marinacci et al. (2011) modeled the effect of such a hot halo on the extraplanar gas kinematics. They found that interaction at the interface between clouds and the hot halo may lead to cooling of gas from the hot gas reservoir and subsequent infall into disks. Fraternali et al. (2013) showed that supernova-driven galactic fountains can transport gas from a hot halo down to the galactic disk. Whether the accretion is from a pre-existing hot gaseous halo or directly from the IGM, the lags we observe may provide indirect evidence for accretion of gas. Since the external gas would have slower rotation than the gas in galactic disks, accretion of significant amounts of the gas will create noticeable lags, i.e., the higher the ratio of infalling-to-local gas, the larger the lag we observe. Although this explanation is plausible, additional observations and simulations are necessary to confirm it.

We performed modeling of the radial velocities and emission line fluxes of the ionized gas provided by MaNGA data analysis pipeline MPL-4. Our models were designed to derive the vertical lag of the rotation velocity of ionized gas that is observed in velocity fields of the gas.

We selected a sample of 67 edge-on galaxies from more than 1400 galaxies observed by MaNGA. In 25 galaxies of this sample (i.e., in 37\%) we observe a regular lag of the rotation curve in the extraplanar gas. We derived the lag in these 25 galaxies, which span a wide range of masses and luminosities.

We observe no correlation between the amplitude of the lag and galactic star formation activity traced by $\mathrm{H} \alpha$ and IR luminosity. At the same time, the lag demonstrates a noticeable correlation with galactic stellar mass, hence dark matter halo mass, and central stellar velocity dispersion. The lags are larger in early-type disk galaxies, whereas smaller lag values are more typical for late-type galaxies. Early-type galaxies are known to be gas-poor, while late-type galaxies contain more gas. We interpret our results as evidence for a higher ratio of infallingto-local gas in early-type galaxies, and in general the data may point at the importance of gas infall in the galactic gas balance.

As the MaNGA observing campaign proceeds, we expect to increase our sample of galaxies with measured lags by a factor of five (given MaNGA observing goals), and to build the largest ever sample of galaxies available for statistical studies of the rotation velocity lags in the extraplanar ionized gas. Accretion from an IGM might not lead to symmetric velocity 
lags, whereas interaction with hot halo gas might. Our future studies will therefore also address in more detail the properties of the galaxies that do not show a regular lag and study the possible difference in the lags above and below disks to distinguish between the different scenarios.

This material is based on work partially supported by the National Science Foundation under Grant No. AST-1615594 to R.A.M.W. A.D. acknowledges support from The Grainger Foundation. D.B. acknowledges support from RSF grants RSCF-14-50-00043. We appreciate the anonymous referee for valuable suggestions that improved the paper.

SDSS-IV acknowledges support and resources from the Center for High-Performance Computing at the University of Utah. The SDSS web site is www.sdss.org.

SDSS-IV is managed by the Astrophysical Research Consortium for the Participating Institutions of the SDSS Collaboration including the Brazilian Participation Group, the Carnegie Institution for Science, Carnegie Mellon University, the Chilean Participation Group, the French Participation Group, Harvard-Smithsonian Center for Astrophysics, Instituto de Astrofísica de Canarias, The Johns Hopkins University, Kavli Institute for the Physics and Mathematics of the Universe (IPMU)/University of Tokyo, Lawrence Berkeley National Laboratory, Leibniz Institut für Astrophysik Potsdam (AIP), Max-Planck-Institut für Astronomie (MPIA Heidelberg), MaxPlanck-Institut für Astrophysik (MPA Garching), Max-PlanckInstitut für Extraterrestrische Physik (MPE), National Astronomical Observatory of China, New Mexico State University, New York University, University of Notre Dame, Observatrio Nacional/MCTI, The Ohio State University, Pennsylvania State University, Shanghai Astronomical Observatory, United Kingdom Participation Group, Universidad Nacional Autónoma de México, University of Arizona, University of Colorado Boulder, University of Oxford, University of Portsmouth, University of Utah, University of Virginia, University of Washington, University of Wisconsin, Vanderbilt University, and Yale University.

\section{References}

Alam, S., Albareti, F. D., Allende Prieto, C., et al. 2015, ApJS, 219, 12 Allen, J. T., Croom, S. M., Konstantopoulos, I. S., et al. 2015, MNRAS, 446, 1567

Baldwin, J. A., Phillips, M. M., \& Terlevich, R. 1981, PASP, 93, 5

Behroozi, P. S., Wechsler, R. H., \& Conroy, C. 2013, ApJ, 770, 57

Benjamin, R. A. 2012, EAS Pub. Ser., 56, 299

Bianchi, S. 2007, A\&A, 471, 765

Binney, J. 2005, in ASP Conf. Ser. 331, Extra-planar Gas Conf., ed. R. Braun (San Francisco, CA: ASP), 131

Bizyaev, D., \& Kajsin, S. 2004, ApJ, 613, 886

Bizyaev, D., \& Mitronova, S. 2002, A\&A, 389, 795

Bizyaev, D., \& Mitronova, S. 2009, ApJ, 702, 1567

Bizyaev, D. V., Kautsch, S. J., Mosenkov, A. V., et al. 2014, ApJ, 787, 24

Blanton, M. R., Bershady, M. A., \& Abolfathi, B. 2017, AJ, submitted (arXiv:1703.00052)

Blanton, M. R., Kazin, E., Muna, D., et al. 2011, AJ, 142, 31

Boettcher, E., Zweibel, E., Gallagher, J. S., III, \& Benjamin, R. 2016, ApJ, 832,118

Bregman, J. N. 1980, ApJ, 236, 577
Brinchmann, J., Charlot, S., White, S. D. M., et al. 2004, MNRAS, 351, 1151 Bundy, K., Bershady, M. A., \& Law, D. R. 2015, ApJ, 798, 7

Collins, J. A., Benjamin, R. A., \& Rand, R. J. 2002, ApJ, 578, 98 de Grijs, R., Peletier, R. F., \& van der Kruit, P. C. 1997, A\&A, 327, 966

Dettmar, R.-J. 1990, A\&A, 232, L15

Dettmar, R.-J. 2004, Ap\&SS, 289, 349

Drory, N., MacDonald, N., Bershady, M. A., et al. 2015, AJ, 149, 77

Fraternali, F., \& Binney, J. J. 2006, MNRAS, 366, 449

Fraternali, F., \& Binney, J. J. 2008, MNRAS, 386, 935

Fraternali, F., Marasco, A., Marinacci, F., \& Binney, J. 2013, ApJ, 764, 21

Fraternali, F., Oosterloo, T., \& Sancisi, R. 2004, A\&A, 424, 485

Gentile, G., Jzsa, G. I. G., Serra, P., et al. 2013, A\&A, 554, 125

Gunn, J. E., Siegmund, W. A., Mannery, E. J., et al. 2006, AJ, 131, 2332

Haffner, L. M., Dettmar, R.-J., Beckman, J. E., et al. 2009, RvMP, 81, 969

Heald, G. H., Rand, R. J., Benjamin, R. A., et al. 2006, ApJ, 636, 181

Heald, G. H., Rand, R. J., Benjamin, R. A., \& Bershady, M. A. 2006, ApJ, 647, 1018

Heald, G. H., Rand, R. J., Benjamin, R. A., \& Bershady, M. A. 2007, ApJ, 663,933

Ho, I.-T., Medling, A. M., Bland-Hawthorn, J., et al. 2016, MNRAS, 457, 1257

Hoopes, C. G., Walterbos, R. A. M., \& Rand, R. J. 1999, ApJ, 522, 669

Hoyle, F., \& Ellis, G. R. A. 1963, AuJPh, 16, 1

Jarrett, T. H., Masci, F., Tsai, C. W., et al. 2013, AJ, 145, 6

Kamphuis, P., Peletier, R. F., Dettmar, R.-J., et al. 2007, A\&A, 468, 951

Kamphuis, P., Peletier, R. F., van der Kruit, P. C., \& Heald, G. H. 2011, MNRAS, 414, 3444

Kamphuis, P., Rand, R. J., \& Jzsa, G. I. G. 2013, MNRAS, 434, 2069

Kylafis, N., Misioritis, A., Papamastorakis, J., \& Xilouris, E. 2001, Ap\&SS, 276, 531

Law, D. R., Cherinka, B., Yan, R., et al. 2016, AJ, 152, 83

Law, D. R., Yan, R., Bershady, M. A., et al. 2015, AJ, 150, 19

Marinacci, F., Fraternali, F., Nipoti, C., et al. 2011, MNRAS, 415, 1534

Matthews, L. D., Gallagher, J. S., \& van Driel, W. 1999, AJ, 118, 2751

Matthews, L. D., \& Wood, K. 2003, AJ, 593, 721

Mosenkov, A. V., Sotnikova, N. Y., Reshetnikov, V. P., et al. 2015, MNRAS, 451, 2376

Nelder, J. A., \& Mead, R. 1965, CompJ, 7, 308313

Norman, C. A., \& Ikeuchi, S. 1989, ApJ, 345, 372

Putman, M. E., Peek, J. E. G., \& Joung, M. R. 2012, ARA\&A, 50, 491

Rand, R. J. 1997, ApJ, 474, 129

Rand, R. J. 2000, ApJL, 537, L13

Rand, R. J., Kulkarni, S. R., \& Hester, J. J. 1990, ApJL, 352, L1

Relaño, M., Lisenfeld, U., Pérez-González, P. G., Vílchez, V. M., \& Battaner, E. 2007, ApJL, 667, L141

Reynolds, R. J. 1971, PhD thesis, Univ. Wisconsin-Madison

Reynolds, R. J., Haffner, L. M., \& Tufte, S. L. 1999, ASP Conf. Ser. 168, New Perspectives on the Interstellar Medium, ed. A. R. Taylor,

T. L. Landecker, \& G. Joncas (San Francisco, CA: ASP), 149

Reynolds, R. J., Scherb, F., \& Roesler, F. L. 1973, ApJ, 185, 869

Rosado, M., Gabbasov, R. F., Repetto, P., et al. 2013, AJ, 145, 135

Rossa, J., \& Dettmar, R.-J. 2003, A\&A, 406, 493

Rossa, J., Dettmar, R.-J., Walterbos, R. A. M., \& Norman, C. A. 2004, AJ, 128,674

Sanchez, S. F., Garcia-Benito, R., Zibetti, S., et al. 2016, A\&A, 594, 36

Shapiro, P. R., \& Field, G. B. 1976, ApJ, 205, 762

Shull, M. J., Jones, J. R., Danforth, C. W., \& Collins, J. A. 2009, ApJ, 699 754

Smee, S. A., Gunn, J. E., Uomoto, A., et al. 2013, AJ, 146, 32

Swaters, R. A., Sancisi, R., \& van der Hulst, J. M. 1997, ApJ, 491, 140

Wright, E. L., Eisenhardt, P. R. M., Mainzer, A. K., et al. 2010, AJ, 140, 1868

Wu, C. J., Walterbos, R. A., Rand, R. J., et al. 2014, in American Astronomical Society, AAS Meeting, 223, 45319

Xilouris, E. M., Byun, Y. I., Kylafis, N. D., et al. 1999, A\&A, 344, 868

Yan, R., Tremonti, C., Bershady, M. A., et al. 2016, AJ, 151, 8

Yoachim, P., \& Dalcanton, J. J. 2006, AJ, 131, 226

Zhu, Y.-N., Wu, H., Cao, C., \& Li, H.-N. 2008, ApJ, 686, 155

Zschaechner, L. K., \& Rand, R. J. 2015, ApJ, 808, 153

Zschaechner, L. K., Rand, R. J., Heald, G. H., et al. 2011, ApJ, 740, 35

Zschaechner, L. K., Rand, R. J., \& Walterbos, R. A. M. 2015, ApJ, 799, 61 